02.5-07 OPTICAL DIFFRACTION AND COMPUTER GENERATED FOURIER TRANSTORM STUDIES OF PARTICLES OF THE POTEX GROUP OF VIRUSES. By P. Tollin, Physics Department, The University, Dundee, Scotland and J.B. Bancroft and J.F. Pichardson, The Faculty of Science, The University of Western Ontario, London, Ontario, Canada.

Five definitive or putative members of the Potex group of viruses have been examined in the electron microscope. They are Cymbidium Mosaic virus, Nerine virus $\mathrm{X}$, Viola Mottle virus, Barrel Cactus virus and Foxtail Mosaic virus, optical diffraction from the electron micrographs reveals that the particles are all helical with a pitch of $3.4-3.5 \mathrm{~mm}$. All the viruses appear to have approximately the same number of subunits per turn, this number is just less than an integer and the integer is most likely to be 9. The only parameter which varies widely for the different particles is the number of subunits in the true repeat and this does not represent substantial structural difference.

The determination of the number of subunits per turn would be helped by a knowledge of the phase of the reflection on the first layer line in the optical diffraction pattern. Two systems, one based on an 'Apple' microcomputer controlled flat-bed microdensitometer and one based on a PDP Il controlled digital camera, have been devised to obtain digital representations of the electron micrographs. The application of a Fast Fourier Transform routine to the data allows the phases of the transforms to be obtained. This process has been applied successfully to particles of RNA-free reconstructed protein subunits and efforts are being made to apply it to the intact virus.

\section{5-08 NEUTRON SCATTERING STUDIES OF HISTONE} COMPLEXES. BY

R. Douglas Carlson and Teresa H. Kelley, Biology Department, Brookhaven National Laboratory, Upton, New York 11973.

The basic unit of chromatin structure is the nucleosome. It consists of a segment of DNA wrapped around a protein core comprised of 2 each of the histones $\mathrm{H} 2 \mathrm{~A}, \mathrm{H} 2 \mathrm{~B}, \mathrm{H} 3$ and $\mathrm{H} 4$. This histone octamer can be dissociated from the DNA in $2 \mathrm{M} \mathrm{NaCl}$ and can be studied in solution at that ionic strength. At lower ionic strength the octamer further dissociates into an $(\mathrm{H} 3-\mathrm{H} 4)_{2}$ tetramer and an $\mathrm{H} 2 \mathrm{~A}-\mathrm{H} 2 \mathrm{~B}$ dimer.

It appears as though these smaller histone complexes exist intact within the larger core octamer, although it is not known what conformational changes may accompany this association. Neutron scattering methods are being used to study this problem. Data are presented here on the solution conformations of these histone complexes. They were isolated by salt extraction at pH7 from chicken $\mathrm{RBC}$ chromatin bound to hydroxyapatite colums, or formed by mixing dilute solutions containing stoichiometric amounts of purified $\mathrm{H} 2 \mathrm{~A}$ and $\mathrm{H} 2 \mathrm{~B}$ or $\mathrm{H} 3$ and $\mathrm{H} 4$. Reconstituted octamer was formed from tetramers and dimers both in the presence and absence of DNA. Radii of gyration, volumes and shapes are discussed and the scattering data are compared with those calculated for model structures. (Supported by the U. S. Dept. of Energy.)
02.5-09 CRYSTALLINE ARRAYS OF LARGE RIBOSOMAL SUBUNITS FROM E. COLI PRODUCED IN VITRO. BY Michael W. Clark and James A. Lake, Molecular Biology Institute and Department of Biology, University of California, Los Angeles, California 90024, U.S.A.

In vitro conditions have been determined for obtaining crystalTine sheets of large ribosomal subunits from $E$. coli. These sheets, slightly curved, close on themselves to form large, $1250 \AA$ diameter tubes that are approximately 12,000 \& 7 ong. They are the first arrays of 1 arge subunits produced in vitro that are suitable for study by three-dimensional reconstruction (helices of small ribosomal subunits have been previously obtained; see Clark et al., J. Mol. Biol. (1979) 135, 507). Large ribosomal subunits remain in solution for extended periods (up to 5 months) during this procedure and their structural integrity as assessed by sucrose gradients and by electron microscopy is preserved.

Electron micrographs of arrays diffract to 45 A resolution. Optical diffraction of electron micrographs of flattened tubes shows that there are two Targe subunits per asymmetric unit in a primitive lattice with plane group symmetry p2 $(a=360 \pm 20 \AA, b=380 \pm 20 \AA, a=$ $\left.118 \pm 5^{\circ}\right)$. This packing can also be referred to a centered lattice $(a=380 \pm 20 \AA, b=660 \pm 20 \AA)$ with approximate symmetry $\mathrm{cmm}$.

Supported by grants from NIH and NSF to JAL.

02.6-01 MOLECULAR CONFORMATION AND PACKING OF MEMBRANE LIPIDS. By S. Abrahamsson, I. Pascher and S. Sundell, Dept. of Structural Chemistry, University of Göteborg, Göteborg, Sweden.

Glycerophospholipids and sphingolipids are important constituents of biological membranes. In order to relate the chemical structure of these Iipids to their structural and functional role in membranes a detailed knowledge of their conformation and interactions is necessary. Accumulated structural data from single crystal analyses of single and double chain Iipids reveal the following features: - The phosphorylcholine and phosphorylethanolamine head
groups adopt a preferred conformation.

- The P-N dipole are parallel to the Iipid bilayer surface.

- The polar head groups have a directional effect on the glycerol backbone.

- The glycerol C-C bond next to the head group provices an axis for independent rotation of the polar head group and the hydrophobic moiety.

- The conformation and orientation of the glycerol dicarboxylic ester group remains the same for different double chain lipids.

- The different head groups show important differences in their interactions and space requirements. The hydrocarbon chains can adopt to the space requirements of the polar group by varying their tilt towards the layer plane. Thereby the chains bend off adjacent to the $\mathrm{sp}^{2}$ hybridized carbonyl carbon atoms.

NMR studies indicate that the characteristic molecular conformation is retained in different environments and states of aggregation. The presented structure investigations show that the phase behaviour and thus the possible functional role of membrane lipids is determined by differences in interaction of the head groups. 